\title{
Planificación estratégica de la gestión de la Sociedad Chilena de Enfermedades Respiratorias en el bienio 2010-2011
}

\author{
Strategic planning for the management of the Chilean Respiratory Disease Society \\ in the biennium 2010-2011
}

Al iniciar la gestión del nuevo directorio de la Sociedad Chilena de Enfermedades Respiratorias que presido y que se extenderá durante los años 2010 y 2011, deseamos compartir con los socios los objetivos que nos hemos propuesto en este periodo. Sin duda llegamos con muchos sueños que esperamos ver materializados al final de nuestra gestión. Sin embargo, debemos concretar algunos aspectos especificos para lo cual hemos elegido el método de la planificación estratégica. El concepto central, es fijar metas con un encargado responsable, idealmente un miembro del directorio, quien deberá formar un equipo de trabajo. Este programa debe ser evaluado periódicamente para analizar el cumplimiento de estas tareas.

A continuación explicaremos las áreas especificas de trabajo a desarrollar.

En primer lugar, debemos mejorar la imagen y prestigio de nuestra Sociedad. Como objetivo especifico debe aumentar la participación de la Sociedad y de sus socios en los medios de comunicación, opinando sobre los temas relevantes de la especialidad. Sin duda debemos estar presentes en días significativos como el día de la EPOC (Enfermedad Pulmonar Obstructiva Crónica), el día del Asma y "el día Sin Fumar". Paralelamente debemos establecer una relación de trabajo con las autoridades del Ministerio de Salud y establecer nexos de colaboración mutua con las Sociedades Latinoamericanas en particular con la Asociación Latinoamericana de Tórax (ALAT).

En segundo lugar, debemos trabajar en el tema de la educación continua. El perfeccionamiento médico continuo, después de realizar la beca de la especialidad hoy en día no es sólo un imperativo ético para el cuidado de los pacientes, sino de vigencia y supervivencia en un mundo cada vez más competitivo y globalizado. Entre los objetivos especificos está la creación de un curso continuo de neumología pediátrica y de adultos vía on-line con una duración de al menos dos años.

Otro curso a planificar es uno de actualización ("State of the Art") anual, esto es un curso de neumología de adultos y otro de neumología pediátrica por separado de un día de duración.

Durante varios años en nuestra Sociedad se realizaron encuentros de especialistas que servían para discutir casos clínicos interesantes de diferentes hospitales y centros de la especialidad. Estas reuniones servían para mantener el contacto entre nosotros y como un puente entre las Jornadas de Otoño, las Jornadas de Invierno y el Congreso. Nos proponemos reestablecerlos con una frecuencia de tres veces al año durante los meses sin actividades tradicionales de la Sociedad.

Un tercer tema relevante es la participación de los neumólogos pediatras en nuestra Sociedad. Uno de los objetivos centrales de esta administración es estimular la admisión y permanencia de estos colegas en nuestra Sociedad. Además, tenemos la intención de crear un comité pediátrico asesor del directorio, con tareas especificas tales como participar en la creación de los programas pediátricos de la Sociedad y establecer una instancia de diálogo y de colaboración mutua con la Sociedad Chilena de Neumología Pediátrica.

En cuarto lugar, debemos crear grupos de trabajo sobre áreas de interés y de actualidad tales como Curriculum de Pre y Post-grado, Guías de Apnea del Sueño, Guías y AUGE en Asma, EPOC, neumonía y Enfermedades Respiratorias Virales. 
En quinto lugar, tenemos una deuda en el tema de comunicación y participación de los socios. Nos hemos propuesto crear un Boletín electrónico disponible en la página web, que sea enviado a todos los socios vía E-mail y la creación de un blog de opinión exclusivo para los miembros de la Sociedad.

En sexto lugar, queremos seguir incentivando la investigación para lo cual podrían mejorarse los fondos especiales para este objetivo, especialmente para becados y post becados inmediatos de la especialidad. Para esto necesitamos también la colaboración de investigadores "senior" o establecidos que acojan y guien a estos investigadores jóvenes.

Finalmente, lo que deseamos construir debe estar basado en los cimientos que establecieron nuestros predecesores. Deseamos invitar a participar a los ex-presidentes en un comité especial para apoyar la gestión de este nuevo directorio y por qué no decirlo a soñar con la Sociedad Chilena de Enfermedades Respiratorias del futuro.

Dr. Fernando Descalzi Muñoz

Presidente,

Sociedad Chilena de Enfermedades Respiratorias 